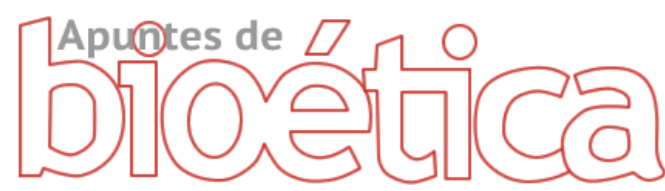

https://doi.org/10.35383/apuntes.v1i1.187

\title{
Fortalecimiento de la ética en la formación de profesionales en odontología desde la perspectiva de la bioética personalista
}

\author{
Vílchez Bellido Dina ${ }^{1}$
}

\section{INFORMACIÓN DEL ARTÍCULO RESUMEN}

Recibido el 26 de setiembre de 2018

Aceptado el 20 de noviembre de 2018

La formación en ética durante la educación universitaria constituye una

Palabras claves:

Ética,

Formación universitaria,

Odontología,

Bioética personalista necesidad para el logro de profesionales comprometidos con su labor, por lo que el desarrollo de estrategias dentro de un enfoque adecuado, que reconozca el carácter ontológico de la persona y su dignidad, permitiría su fortalecimiento desde las universidades. La presente investigación tiene como objetivo presentar los fundamentos que permitirán definir posteriormente las estrategias de fortalecimiento de la ética en la formación de profesionales en Odontología, desde la perspectiva de la Bioética Personalista. Se desarrolló una investigación descriptivo-documental, que incluyó el análisis de la problemática actual de la ética en la formación de profesionales en odontología, considerando la revisión de documentos y contenidos curriculares de cuatro universidades locales; se presentaron las bases teóricas de la ética en la formación universitaria y se exploró la perspectiva de la Bioética Personalista. Se concluye que la ética debería desarrollarse considerando estrategias que contemplen una presencia transversal de ésta a lo largo del plan de estudios, con una visión desde la primera persona y dentro de una bioética no relativista, como la enfocada por la bioética personalista, cuyas bases filosóficas y antropológicas permitan consolidar el desarrollo de la ética durante la formación universitaria.

${ }^{1}$ Docente Universitario Universidad Wiener, Cirujano dentista. Email: divibe@hotmail.com. ORCID: https://orcid.org/0000-0003-2675-5084 


\section{Strengthening ethics in the training of dental professionals from the perspective of personal bioethics}

\section{ABSTRACT}

\section{Keywords:}

Ethics,

University education,

Dentistry,

Personalist bioethics
Training in ethics during university education is a necessity for the achievement of professionals committed to their work, so the development of strategies within an appropriate approach, which recognizes the ontological character of the person and his dignity, would allow its strengthening from the universities. The objective of this communication is to present the fundamentals that will later define the strategies for strengthening ethics in the training of professionals in dentistry, from the perspective of Personalist Bioethics. A descriptive-documentary research was developed, which included the analysis of the current problems of ethics in the training of professionals in dentistry, considering the revision of documents and curricular contents of four local universities; the theoretical bases of ethics in university training were presented and the perspective of Personalist Bioethics was explored. It is concluded that ethics should be developed considering strategies that contemplate a transversal presence of ethics throughout the curriculum, with a vision from the first person and within a non-relativist bioethics, such as that focused on personal bioethics, whose philosophical and anthropological bases allow consolidating the development of ethics during university education

\section{Introducción}

La Ley General de Educación 28044 (2003) establece que la educación peruana debe considerar a la persona como centro y agente fundamental de la formación educativa, teniendo a la ética como sustento, para lograr la trasformación de la sociedad y el desarrollo del país.

Pese a ello, el enfoque ético actual en la formación profesional es reducido, y los resultados evidencian un quehacer profesional que desconoce los principios éticos.

La realidad social imperante conduce a cuestionar el perfil ético de los currículos universitarios, que al parecer no logran su cometido de formar profesionales que legitimen a la misma profesión a través del bien y la utilidad que deben proporcionar a la sociedad. (Díaz, Pérez y Lara, 2016, p.44)

Esta problemática llama al desarrollo de estrategias de fortalecimiento de la ética en las universidades que permitan una formación profesional idónea.

La presente investigación busca resolver el problema: ¿Cuáles serán las estrategias de fortalecimiento de la ética en la formación de profesionales en odontología desde la perspectiva de la Bioética Personalista? Para ello, en esta comunicación, se realiza un análisis de la problemática actual de la ética en la formación de profesionales en odontología, considerando la revisión de documentos y contenidos curriculares de cuatro universidades locales; se 
presentan además, las bases teóricas de la ética en la formación universitaria, y se explora la perspectiva de la Bioética Personalista; todo ello nos brindará el fundamento necesario para el establecimiento de estrategias de fortalecimiento de la ética.

La necesidad de definir estrategias de fortalecimiento de la ética en la formación profesional en odontología desde la Bioética Personalista, se justifica ante la necesidad de formar profesionales odontólogos que posean habilidades para el análisis y resolución de los dilemas éticos que se le presenten en su práctica diaria (Puccinelli, 2013).

Se sigue un enfoque antropológico personalista fundamental, que busca el bien integral de la persona (López, 2013). Su diseño e implementación permitirá una transformación significativa del comportamiento bioético clínico de los estudiantes (Domínguez, 2014). La principal beneficiaria será la comunidad universitaria.

\section{Metodología}

La presente investigación adoptó un paradigma teórico y un enfoque cualitativo (Hernández, 2014)².

El tipo de investigación fue de tipo descriptivo-documental (Bernal, 2010) ${ }^{3}$.

Las técnicas empleadas fueron el fichaje y el análisis documental. Se registró en fichas los

\footnotetext{
${ }^{2}$ La investigación cualitativa permite, siguiendo una lógica y un proceso inductivo, la obtención de datos a través de evidencias verbales, audiovisuales y textuales, en búsqueda de un mayor entendimiento del tema de investigación; estos datos posteriormente son analizados e interpretados por medio de la descripción de la información y el desarrollo de temas.

${ }^{3}$ La investigación descriptiva nos ayuda a identificar las características del fenómeno a estudiar; y es por medio de la investigación tipo documental que se analiza la información disponible sobre el fenómeno estudiado a través de la consulta de fuentes documentales escritas, documentos fílmicos y grabados.
}

datos de libros, revistas científicas e información de buscadores y bases de datos, revistas online de bioética y repositorios de tesis. Se realizó un análisis documental de los textos y resúmenes, así como de documentos académicos y planes curriculares de cuatro universidades locales que desarrollan la carrera de Odontología en pregrado.

Consideramos como criterios éticos, el respeto a las personas y la búsqueda del bien común y la justicia; y como criterios de rigor científico, la claridad, la credibilidad y la profundidad de la información recolectada.

\section{Resultados, análisis y discusión}

La formación en ética durante la educación universitaria constituye una necesidad para el logro de profesionales comprometidos con su labor.

El Reglamento de la Ley de trabajo del cirujano dentista 27878 (2005) reconoce la formación universitaria de los profesionales en odontología, que lo prepara para prestar sus servicios en forma humanística, científica y técnica, en los procesos de promoción, prevención, recuperación y rehabilitación de la salud; interactuando con la persona, la familia y la comunidad, bajo consideraciones éticas, morales y legales.

El Código de Ética y Deontología del Colegio Odontológico Del Perú (2016) señala que en el ejercicio de la profesión odontológica, se respetarán todos los derechos de la persona humana, actuando con veracidad, libertad, justicia y solidaridad. Para esto se debe contar con bases éticas sólidas, adquiridas en la vida familiar, pero consolidadas en la universidad, 
que tiene ante ello un papel decisivo (Díaz, 2015).

La Ley Universitaria 30220 (2014) define a las universidades como "una comunidad académica orientada a la investigación y a la docencia, que brinda una formación humanista, científica y tecnológica con una clara conciencia de nuestro país como realidad multicultural". Esta formación debe desarrollar un enfoque ético.

La formación odontológica debe considerar las características propias de la Odontología.

Una profesión con un profundo sentido social que persigue solucionar los problemas de salud bucal de la población y mejorar su calidad de vida, (...) para conseguir esto, es absolutamente necesario un comportamiento ético-profesional y una responsabilidad social por parte de quienes ejercen la profesión, por lo que estos aspectos valóricos deben ser una preocupación constante y estar en el centro de la formación odontológica que entregan las universidades. (Alcota, Ruiz de Gauna y González, 2016, p. 175)

Se reconoce la necesidad de fortalecer la formación universitaria en ética, que permita un idóneo desempeño de la profesión odontológica.

La educación universitaria busca la formación en conocimientos y destrezas que no puede estar separada de la formación ética profesional. Se busca profesionales competentes para el campo laboral o la investigación; pero, si sólo se pretende esto, la misión de las universidades quedaría reducida a una perspectiva de racionalidad instrumental en la educación de los aspectos cognitivos y técnicos de los profesionales, dejando de lado el sentido humano y social de toda profesión (Alcota et al, 2016). Es necesario que los estudiantes se formen integralmente.
La finalidad de la educación es "formar personas capaces de lograr su realización ética, intelectual, artística, cultural, afectiva, física, espiritual y religiosa, promoviendo la formación y consolidación de su identidad y autoestima y su integración adecuada y crítica a la sociedad" (Ley General de Educación 28044, 2003). En el marco de la Ley Universitaria, se han desarrollado políticas (Ministerio de Educación [MINEDU], 2015) ${ }^{4}$ y sistemas (Sistema nacional de evaluación, acreditación y certificación de la calidad educativa [SINEACE], 2016) a garantizar y velar por la Calidad de la educación universitaria, bajo los principios de justicia, ética, solidaridad y respeto por la diferencia (MINEDU, 2015). La calidad en educación debe entenderse como una formación integral del estudiante que le permita desenvolverse en su accionar bajo principios éticos.

En nuestro país, existen 33 instituciones universitarias que desarrollan la carrera de odontología o estomatología. (Colegio

Odontológico del Perú [COP], 2015) Como parte de esta investigación, se revisaron los contenidos curriculares de cuatro escuelas y facultades de odontología de universidades del medio local. Se escogió la carrera de Odontología en cuatro universidades, considerando la antigüedad (tres de ellas), representatividad (dos estatales y dos particulares): Universidad Nacional Mayor de San Marcos (UNMSM), Universidad Nacional Federico Villarreal (UNFV), Universidad Peruana Cayetano

\footnotetext{
${ }^{4}$ En el 2015, el Estado peruano elabora la Política de Aseguramiento de la Calidad de la Educación Superior Universitaria, que busca garantizar que los servicios educativos universitarios sean de calidad, con una formación integral y de perfeccionamiento continuo, proyectado a lograr un desempeño profesional competente y con valores ciudadanos incorporados.

${ }^{5}$ En el 2016, se publicó un modelo de Acreditación para Programas de Estudios de Educación Superior Universitaria, que señala que en la formación de la persona se debe cuestionar qué tipo de personas se quiere formar y qué tipo de sociedad se aspira alcanzar como producto del proceso educativo.
} 
Heredia (UPCH) y Universidad Norbert Wiener (UNW).

La UNMSM contempla en su misión valores como la ética (Facultad de Odontología de la Universidad Nacional Mayor de San Marcos, 2017). La UNFV considera en su misión, la formación humanística de profesionales (Facultad de Odontología de la Universidad Nacional Federico Villarreal, 2012-2013). La UPCH incluye dentro de las competencias del perfil del egresado, demostrar un comportamiento y compromiso ético, como parte de la misión de formación integral de profesionales "centrada en la persona", con principios científicos, humanísticos y éticos (Facultad de Estomatología de la Universidad Peruana Cayetano Heredia, 2017). La UNW tiene como misión la formación profesional $y$ humanística, y la calidad como un imperativo ético (Universidad Norbert Wiener Odontología, 2018). Encontramos que todas mencionan a la ética, como parte de la información académica que ofrecen, referente a misión, valores, perfil del egresado, objetivos de la carrera y políticas de calidad.

Al explorar sus planes de estudios o mallas curriculares, se hizo evidente el componente ético, pero plasmado sólo en el desarrollo de una asignatura y en diferentes momentos. Así, en la UNMSM se enseña Odontología Ética y Deontología, en el tercer semestre del plan de estudios, con 3 horas semanales (Facultad de Odontología de la Universidad Nacional Mayor de San Marcos, 2018). En la UNFV se desarrolla Odontología Legal y Deontología en el quinto año, con 4 horas semanales (Facultad de Odontología de la Universidad Nacional Federico Villarreal, 2017). En la UPCH se dicta Odontología Legal y Forense en el sexto ciclo (Facultad de Estomatología de la Universidad Peruana Cayetano Heredia, 2016). Y en la UNW se incluye a Ética y Responsabilidad Social, en el cuarto ciclo, con 3 horas semanales (Universidad Norbert Wiener Odontología, 2018). Observamos como los términos empleados para designar la asignatura variaron en su nominación; algunas sí incluían el término ética, mientras otras lo designaban con términos referentes al aspecto normativo de ella, debiendo permanecer ambas temáticas asociadas para guiar el desempeño de la profesión.

En nuestra investigación, respecto al momento en que se desarrollan estas asignaturas, encontramos que mientras dos universidades las incluyen a los inicios de la carrera, otra lo hace a la mitad y la otra en el último año de carrera. Esta diferencia parece caracterizar los ambientes universitarios. La enseñanza de la ética está presente en las mallas curriculares, pero queda aún por establecer su adecuado desarrollo didáctico, notándose que priman más las asignaturas desarrolladas como sistema moral y deontológico, con carácter informativo y con motivaciones de naturaleza normativa (Guerrero y Gómez, 2013).

Es fundamental el estudio de la ética desde los primeros años de formación de la conducta moral de los estudiantes universitarios, pero también es pertinente su enseñanza en los últimos años, considerando su inclusión en asignaturas independientes y de manera interdisciplinaria. Existen experiencias de enseñanza de ética en los primeros años, que se complementa posteriormente con la práctica clínica en los últimos años, con la formación de pequeños grupos de análisis y acompañamiento, enfocando la problemática previamente estudiada en la teoría. Esta integración podría lograrse, al incorporar los contenidos éticos en el currículo de manera transversal (Villegas, 2015).

La ética, como tema transversal, hace que el aprendizaje sea significativo y trascendente, en que los estudiantes se hacen conscientes de la 
necesidad de la práctica de una libertad ética y socialmente responsable (Puccinelli, 2013).

El carácter transversal de una carrera universitaria se orienta a la inclusión de contenidos en varias asignaturas de manera complementaria Esta experiencia de incorporación de la ética, como un eje transversal, ya se desarrolla en nuestro país (Puccinelli, 2013) ${ }^{6}$.

La formación ética de profesionales de la salud "es una necesidad intrínseca que no debe estar sujeta a un marco axiológico (...) y será una exigencia ya no solamente de una manera escolarizada como asignaturas específicas sino de manera transversal" (Yacarini, 2014, p. 588). Se reafirma la necesidad del desarrollo transversal de la ética en la formación universitaria.

En la actualidad se hace difícil que los contenidos éticos en las mallas curriculares se orienten a una educación de la acción moral de los estudiantes universitarios, caracterizadas por la tendencia al utilitarismo y al libertarismo (Guerrero y Gómez, 2013).

La inclusión de la ética debe ser de una manera gradual, en contenidos y en momentos cursados, de tal modo que acompañe a los alumnos en las asignaturas a lo largo de todo el currículo, proponiendo su enseñanza tanto en los primeros semestres, como fundamento, y en los últimos, como apoyo al desempeño clínico odontológico de los alumnos (Cortés, Meneses, Ortiz y Balderas, 2014).

El resultado de lo expuesto coloca a la ética como una temática que cuenta con un mínimo espacio de desarrollo en los planes de estudio

\footnotetext{
${ }^{6}$ Se hace referencia a la Escuela de Odontología de la Universidad Católica Santo Toribio de Mogrovejo, en que se introduce la enseñanza de la Bioética de manera transversal, durante los 12 semestres académicos.
}

universitarios ${ }^{7}, \quad$ reduciéndose a la implementación de una asignatura, muchas veces insuficiente, y no complementada por la práctica. La ética, como eje de la formación profesional, debería tener una presencia transversal a lo largo de todo el plan de estudios. Creemos importante considerar la ética desde el inicio de la carrera universitaria, sentando las bases éticas de la formación profesional, y permanecer hasta los últimos años, en que el estudiante empieza a desarrollar su práctica profesional.

La ética y la bioética no normativizan todo, sino que ofrecen criterios generales para que las personas tomen decisiones pertinentes ante determinadas situaciones. La ética orienta la dirección de la formación universitaria hacia el bien, pero en la práctica tiene diferentes enfoques adoptados, dentro de tradiciones, corrientes y modelos (De Mestral, 2014).

Existen dos tradiciones éticas: la latina que se fundamenta en la ética griega, reforzada por la cultura cristiana y consta de normas absolutas y heterónomas; y la anglosajona, basada en un lenguaje de lo correcto y lo incorrecto, el del derecho y el deber (De Mestral, 2014).

Entre las corrientes y modelos en ética, estos difieren según los valores y los principios que los caracterizan, existiendo las éticas consecuencialistas, que a través de la deliberación se valora las acciones por sus resultados para el mayor número de personas; las éticas deontologistas en que las consecuencias de las acciones no determinan su bondad, con normas que limitan nuestro actuar, considerando la dignidad humana como absoluta e irrenunciable y el bien moral como

\footnotetext{
${ }^{7}$ En el plan operativo de la carrera de Odontología de la UNMSM, la asignatura de Odontología Ética y Deontología pertenece al área curricular de Comportamiento ético y gestión de la salud, que ocupa sólo el $1.93 \%$ del total de créditos del plan curricular y está orientada a fortalecer la conducta ética durante la atención de los pacientes.
} 
afirmación de la dignidad, con valores absolutos, como la ética personalista; y el principialismo, que plantea resolver los conflictos basándose en unos principios éticos universales, beneficencia, no maleficencia, autonomía, y justicia, pero que presentan conflictos al aplicarlos a la práctica médica (De Mestral, 2014).

Se podría definir la ética como un saber filosófico que direcciona la conducta hacia el bien perfecto o fin último de la persona. Mientras la ética estudia los actos humanos libres, los hábitos y las consecuencias morales de las acciones, la moral se encarga del gobierno de la propia conducta, de manera libre, siendo la persona la que proyecta y regula, y no el instinto o la necesidad. El estudio de la ética y la moral tiene una importancia trascendental para dar sentido al actuar personal y profesional, y encamina al hombre hacia la excelencia (De Mestral, 2014).

Podemos considerar dos enfoques de la ética en la formación profesional universitaria; uno desde el punto de vista de la tercera persona o ética normativa, en que la ética se elabora desde el punto de vista del observador externo, con un planteamiento más jurídico que ético, marcado por el normativismo y por el legalismo, donde la ética es considerada como un conjunto de normas y la moral un manual de instrucciones, de corte relativista y en que por consenso se establece la verdad; y el otro desde el punto de vista de la primera persona o ética de la virtud, donde la ética surge de una reflexión interior de la conciencia como un intento de iluminar con la razón práctica las decisiones a tomar y en el que la educación es una actividad liberadora, de conocimiento de la verdad y que pone el bien como meta de conducta. (Echeverría, 2013).

Mientras para la ética normativa, no interesa cómo se ha llegado a tomar una decisión y sólo importa el hecho que hubo una acción y si ésta es correcta o incorrecta, para la ética de la virtud se buscan resultados concretos en el ámbito social, es decir lograr que un sujeto sea más virtuoso y libre, un mejor ciudadano que asume responsabilidades $y$ es solidario. EI docente de ética, para la ética de la tercera persona, sólo define la licitud de una acción (permitida o prohibida), y sus lecciones se limitan a examinar casos, circunstancias, objetos, fines, agravantes y atenuantes, sin considerar lo relacionado al tipo de persona que se quiere ser al actuar de una manera determinada; mientras que, desde la ética de la primera persona, el docente de ética es un motivador óptimo, que confía en que puede potenciar las características de sus alumnos, educando ciudadanos solidarios y que sabe conducir, aconsejar, prevenir y acompañar al alumno por el camino de la adquisición de virtudes (Echeverría, 2013).

Si la ética sólo se centra en lo normativo y el consenso, dejaría de direccionar la conducta hacia el bien; "de nada vale una educación basada en la transmisión de conocimientos eruditos o en un simple adiestramiento, si no se presta especial atención a dirigir hacia el bien la energía operativa del sujeto" (Echeverría, 2013, p. 156).

Se debe tener claro que "educar a la persona para que module su acción con sujeción a normas y a juicios morales, no equivale a enseñarle a filosofar sobre la moral" (Guerrero y Gómez, 2013, p. 124). Consideramos el enfoque desde el punto de vista de la primera persona necesario para la formación ética universitaria, que contribuya a su formación como persona, y en la que el docente interviene activamente encaminando al estudiante para su realización personal y profesional, como sujeto libre y virtuoso. 
La bioética podría definirse como el "diálogo reflexivo entre las ciencias biológicas y la ética" (De Mestral, 2014, p. 50), en que el valor de la vida estará siempre primero frente a los avances tecnológicos. La inserción de la bioética en el campo educativo debe ser considerada, pues las universidades, en su papel de formadoras de profesionales e investigadores, enfrentan permanentemente el desafío de cuestiones del impacto de la intervención tecnocientífica en la vida. (Villegas, 2015).

La bioética debe constituirse como la conciencia crítica de la civilización tecnológica, que tiene como pilares la conexión entre la ética y la ciencia y la distinción entre progreso humano y desarrollo tecnológico, y que necesita una base teórica antropológica y ética para lograr ello. Se plantea la importancia de un análisis bioético en el ambiente universitario de las ciencias de la salud que considere desde el tipo de personas que se quiere formar, la incorporación o profundización de contenidos éticos de manera transversal en el diseño curricular y una adecuada metodología (Yacarini, 2014, p. 587).

En la formación ética universitaria consideramos dos corrientes de la Bioética, la Bioética relativista o laicista, en que la dignidad del ser humano queda reducida sólo a la autonomía de la voluntad y al libre desarrollo de la personalidad del individuo y que demanda la emancipación de cualquier orden moral, situando su fundamento en el consenso; y la Bioética no relativista u objetivista, que considera la dignidad humana como principio ético y jurídico fundamental, así como los derechos y deberes que se derivan a partir de ella (López, 2013).

Dentro del enfoque relativista se situaría la Bioética principialista, que se desarrolla en torno a los principios clásicos de autonomía, beneficencia, no maleficencia y justicia, y que a pesar de ser considerados como principios absolutos, presentarían deficiencias y carencias, como que no tiene claro qué se entiende, por bien de la persona y autonomía del individuo (López, 2013).

Algunos procesos educativos se desarrollan en torno a la Bioética principialista. Villegas (2015) considera la formación en Bioética en el área de salud debe tener de referente los principios de corte principialista, sin dejar de lado el reconocimiento de la dignidad humana. Domínguez (2014) también recalca la importancia de ser un individuo ético y bioético, que va más allá de la moralidad, y que la bioética se constituye como una ciencia aplicada a la odontología, teniendo como pilares del comportamiento bioético clínico en las instituciones universitarias a los principios del principialismo.

En cambio, en el enfoque no relativista se desarrolla la Bioética personalista, con sus principios de la vida física, principio de totalidad o terapéutico, principio de libertad y responsabilidad, y principio de sociabilidad y subsidiariedad, que están vinculados entre sí mediante una antropología personalista fundamental en la búsqueda del bien integral de la persona (López, 2013); cuyo principal promotor es Elio Sgreccia.

Es la bioética personalista que toma de referencia el valor fundamental de la vida humana, la trascendencia de la persona, la concepción integral de la persona y la relación de prioridad y complementariedad entre persona y sociedad (Sgreccia, 2014). El centro del interés de la bioética personalista lo constituye la persona, definida como una unidad de cuerpo y espíritu, que se constituye como "ser digno y anhelante de significado" (De Mestral, 2014, p. 31). Se evidencia la relevancia de reconocer la 
dignidad humana de la persona, inherente a ella y que le da sentido a su vida.

La práctica odontológica debe desarrollarse bajo principios éticos y bioéticos que permitan un buen actuar frente a la atención de personas (pacientes); es necesario pues "formular una base ética alcanzable, que sustente el suministro de un servicio de sanidad bucal de alta calidad" (Izzeddin, Spina y Tosta, 2010, p. 209).

Al respecto, la Asociación Dental Americana, en 1992, presentó los Principios de Ética y Código de Conducta Profesional, como guía al desempeño de la profesión odontológica, destacando entre ellos, el reconocimiento a la persona y su dignidad, un trato igualitario, justo y solidario, y una práctica odontológica orientada bajo principios éticos, científicos y legales. Sugiere que el personal dedicado a la salud bucal debe estar comprometido con principios bioéticos como los de la bioética personalista, y que la práctica odontológica sea entendida como una profesión científica basada en los valores universales del ser humano (Izzeddin et al, 2010).

La situación actual de la ética y de la bioética en los ambientes universitarios lleva a plantearse si se está haciendo lo correcto en su enseñanza-aprendizaje. "EI problema surge cuando todo el proceso formativo se articula en torno a los casos prácticos, con una carencia de presupuestos filosóficos y antropológicos que convierte en estéril y relativa cualquier discusión o debate" (López, 2013, p. 88). Es necesario elaborar estrategias para fortalecer la ética en la formación universitaria, con un adecuado enfoque, como el de la Bioética personalista, que tenga como base una visión antropológica y conceptualizando lo moral, como un conjunto de normas objetivas y universales.
Los estudiantes universitarios necesitan una preparación con base ética para que asuman su papel en la sociedad. "La educación ética constituye la primera responsabilidad social de la universidad y de los docentes universitarios" (Echeverría-Falla, 2013). La enseñanza de la ética y de la educación moral a nivel universitario debe considerar el nivel de maduración de juicio moral de los estudiantes, pero guiada de estrategias didácticas universitarias idóneas para consolidar estructuras universales de juicio moral con el desarrollo de programas de formación pedagógica docente (Guerrero y Gómez, 2013).

Se hace necesaria una formación ética universitaria con base en el reconocimiento de la dignidad de la persona, desde una perspectiva no relativista y con un enfoque bioético personalista.

\section{Conclusiones}

La ética, como eje de formación profesional, debe tener una presencia transversal en el plan de estudios, considerando a la dignidad humana como valor fundamental.

Desde el inicio de la carrera universitaria deben sentarse las bases éticas de la formación profesional, las que deben aplicarse en su práctica profesional universitaria.

El enfoque de la ética, desde el punto de vista de la primera persona y dentro de una bioética no relativista, se constituye como el mejor referente para la formación ética en el ámbito universitario odontológico, en el que el docente universitario intervenga activamente y encamine al estudiante en una formación en virtudes y un crecimiento como persona. 
Se debe considerar el desarrollo de estrategias de fortalecimiento de la ética, desde la perspectiva de la bioética personalista, cuyas bases filosóficas y antropológicas permitan consolidar el desarrollo de la ética durante la formación universitaria.

\section{Recomendaciones}

Se recomienda continuar con la elaboración de estrategias de fortalecimiento de la ética en la formación universitaria, manteniendo la perspectiva de la bioética personalista, considerando sus bases filosóficas y antropológicas.

\section{Bibliografía}

Alcota, M., Ruiz de Gauna, P., y González, F. (2016). El profesionalismo en la formación odontológica. Rev Fac Odontol Univ Antioq, 28(1), 158-78.

Bernal, C. (2010). Metodología de la investigación, Colombia: Pearson.

Colegio Odontológico del Perú. (2016) Código de Ética y Deontología. Lima, Perú: COP.

Colegio Odontológico del Perú. (2015). Directorio de facultades de Odontología. Recuperado de http://www.cop.org.pe/directorio-defacultades-de-odontologia.

Cortes V., Meneses M., Ortiz J, y Balderas C. (2014). Los programas de formación ética en Odontología. Propuestas para que la ética tenga más impacto en la licenciatura de Cirujano Dentista. Educación y Salud
Boletín Científico de Ciencias de la Salud del ICSA, 3(5).

De Mestral, E. (2014). Manual de Bioética. Asunción, Paraguay: Facultad de Ciencias Médicas Universidad Nacional de Asunción.

Díaz F., Pérez M. y Lara Y. (2016). Para enseñar ética profesional no basta con una asignatura: Los estudiantes de Psicología reportan incidentes críticos en aulas y escenarios reales. Revista Iberoamericana de Educación Superior, 7(18), 42-58.

Díaz, S. (2015). Práctica de la ética en el desarrollo formativo de los estudiantes de una universidad privada de Chiclayo (tesis de maestría). Universidad Católica Santo Toribio de Mogrovejo, Chiclayo, Perú.

Domínguez, M. (2014). Optimizando el comportamiento bioético clínico de estudiantes de odontología (tesis de maestría). Universidad de Carabobo, Venezuela.

Echeverría C. (2013). Educación ética: inormas o virtudes? ¿Qué giro debe tomar la enseñanza de la ética en la formación de universitarios solidarios? Persona $y$ Bioética, 17(2), 151-67.

Facultad de Odontología de la Universidad Nacional Mayor de San Marcos. (2017). Misión, Visión, Valores. Facultad de Odontología. Escuela profesional de odontología de pregrado. Recuperado de https://odontologia.unmsm.edu.pe/nosotr os/.

Facultad de Odontología de la Universidad Nacional Federico Villarreal. (20122013). Misión, visión y valores. 
Recuperado de http://www.unfv.edu.pe/facultades/fo/nue stra-facultad/mision-vision-valores.

Facultad de Estomatología de la Universidad Peruana Cayetano Heredia. (2017). Perfil del egresado, Misión, Visión. Recuperado de

http://www.upch.edu.pe/faest/egresado/ mision-vision

Facultad de Odontología de la Universidad Nacional Mayor de San Marcos. (2018) Plan de estudios 2018, Facultad de Odontología, Escuela profesional de odontología de pregrado. Recuperado de https://odontologia.unmsm.edu.pe/escuel a-profesional/.

Universidad Norbert Wiener Odontología. (2018). Perfil de la carrera, plan curricular Recuperado de http://www.uwiener.edu.pe/pregrado/carr era-de-odontologia/.

Facultad de Estomatología de la Universidad Peruana Cayetano Heredia. (2016). Plan curricular. Recuperado de http://www.upch.edu.pe/faest/images/sto ries/direccion_carrera/plan_2016_2020.jp g.

Facultad de Odontología de la Universidad Nacional Federico Villarreal. (2017) Guía del Estudiante-Odontología-UNFV 2017. Recuperado de http://www.unfv.edu.pe/facultades/fo/ima ges/2017/Guia_Estudiante/Guia_Estudiant e_2017.pdf.

Guerrero M., y Gómez D. (2013). Enseñanza de la ética y la educación moral, ipermanecen ausentes de los programas universitarios? Revista Electrónica de Investigación Educativa, 15(1), 122- 35.

Hernández R. (2014). Metodología de la investigación. México: Mc Graw Hill Education.

Izzeddin R., Spina, M., y Tosta E. (2010). Dimensión ética en la práctica odontológica, Visión personalista. Acta Bioethica, 16(2), 207-10.

Ley 28044: Ley general de educación. Diario Oficial El Peruano, Lima, Perú, 28 de julio de 2003.

Ley 30220: Ley universitaria, Diario Oficial EI Peruano, Lima, Perú, 9 de julio de 2014.

López J. (2013). La bioética personalista en los planes de estudio universitarios. Cuadernos de bioética, 24(1), 79-90.

Ministerio de Educación (2015). Política de aseguramiento de la calidad de la educación superior universitaria, Lima, Perú: MINEDU.

Puccinelli M. (2013). Experiencia en la enseñanza de la Bioética en la Escuela de Odontología en una universidad local (tesis de maestría). Universidad Católica Santo Toribio de Mogrovejo, Chiclayo, Perú.

Reglamento de Ley de trabajo del cirujano dentista 27878. Diario Oficial El Peruano, Lima, Perú, 25 de julio de 2005.

Sgreccia, E. (2014) Manual de Bioética: Fundamentos y ética biomédica. Madrid, España: Biblioteca de Autores Cristianos. 
Sistema Nacional de Evaluación, Acreditación y Certificación de la Calidad Educativa. (2016). Modelo de Acreditación para Programas de Estudios de Educación Superior Universitaria Octubre 2016. Lima, Perú: SINEACE.

Universidad Norbert Wiener Odontología. (2018). La Universidad. Recuperado de http://www.uwiener.edu.pe/somos/.

Villegas E. (2015). Tendencias de la formación bioética en los currículos universitarios. Revista Colombiana de Bioética. 6(1), 103-24.

Yacarini, A. (2014). Experiencia universitaria de la formación en bioética en las Ciencias de la Salud. En León F., León R., y Navarrete V. (Ed.). Bioética para la toma de decisiones, Parte 2. Santiago de Chile, Chile: FELAIBE. 